\title{
Influence of Structure and Microstructure on Deformation Localization and Crack Growth in NiTi Shape Memory Alloys
}

\author{
Partha P. Paul ${ }^{1} \cdot$ Margaret Fortman $^{2} \cdot$ Harshad M. Paranjape ${ }^{3} \cdot$ Peter M. Anderson $^{4}$. \\ Aaron P. Stebner ${ }^{3} \cdot$ L. Catherine Brinson ${ }^{5}$
}

Published online: 12 April 2018

(C) ASM International 2018

\begin{abstract}
Porous NiTi shape memory alloys have applications in the biomedical and aerospace fields. Recent developments in metal additive manufacturing have made fabrication of near-net-shape porous products with complicated geometries feasible. There have also been developments in tailoring site-specific microstructures in metals using additive manufacturing. Inspired by these developments, we explore two related mechanistic phenomena in a simplified representation of porous shape memory alloys. First, we computationally elucidate the connection between pore geometry, stress concentration around pores, grain orientation, and strain-band formation during tensile loading of NiTi. Using this, we present a method to engineer local crystal orientations to mitigate the stress concentrations around the pores. Second, we experimentally document the growth of cracks around pores in a cyclically loaded superelastic NiTi specimen. In the areas of stress concentration around holes, cracks are seen to grow in large grains with [ $\left[\begin{array}{lll}1 & 1 & 0\end{array}\right]$ oriented along the tensile axis. This combined work shows the potential of local microstructural engineering in reducing stress
\end{abstract}

Partha P. Paul

parthapaul2018@u.northwestern.edu

1 Mechanical Engineering, Northwestern University, Evanston, IL 60201, USA

2 Department of Physics, Lake Forest College, Lake Forest, IL 60045, USA

3 Mechanical Engineering, Colorado School of Mines, Golden, CO 80401, USA

4 Materials Science and Engineering, The Ohio State University, Columbus, $\mathrm{OH} 43210$, USA

5 Mechanical Engineering and Materials Science, Duke University, Durham, NC 27708, USA concentration and increasing resistance to propagation of cracks in porous SMAs, potentially increasing the fatigue life of porous SMA components.

Keywords Nickel titanium - Shape memory alloys · Electron back scattering diffraction - Crystal plasticity . Additive manufacturing

\section{Introduction}

Due to their unique property of superelasticity, NiTi shape memory alloys (SMAs) have received significant attention from the biomedical and aerospace industries [1, 2]. In the implant industry, porous NiTi SMAs in particular are a candidate material for bone implants [3]. Porosity can promote tissue growth, and by varying the pore volume fraction, the stiffness of the implant can be matched with the stiffness of the bone. The emerging techniques of metal additive manufacturing (AM) are particularly suitable for making such components due to the ability of these methods to produce near-net-shape products with complicated geometries $[4,5]$.

There is another, less obvious advantage of using metal AM to make porous SMA products or SMA assemblies with microscale features such as holes, channels, or notches. The stress and strain distribution in such assemblies with structural features can be highly heterogeneous, owing to the stress concentrations near the pores [6]. In addition, the deformation in SMAs is highly orientation dependent. Since implants typically undergo cyclic loading, deformation can accumulate over time in these stress-concentration zones and eventually lead to failure. Hence, tailoring local grain orientations around pores or other features may provide a method to relieve stresses, reducing the 
likelihood of crack nucleation around pores. To this end, $\mathrm{AM}$ has the unique flexibility to allow such microstructural tailoring. The control of grain size in AM components of Ti-6Al-4V is possible to some extent by controlling laser parameters such as scan speed and power in laser-based AM builds [7]. More recent efforts have also demonstrated that metal AM can be used to tailor site-specific properties, including grain orientation and morphology [8]. DeHoff et al. showed the possibility of such site-specific orientation control in Inconel 718 by varying electron beam scan parameters in specific patterns [9]. When selective laser melting (SLM) of random-textured raw material is used to build NiTi SMA parts, $\left[\begin{array}{lll}1 & 0 & 0\end{array}\right]$ is observed to orient along the build direction under the laser spot [10]. Franco et al. showed that by controlling the SLM-processing parameters, a spatial variation in the transformation temperatures can be obtained in a NiTi SMA build [11]. Wang et al. recently studied the relationship between laser parameters in SLM and the local composition and transformation temperatures, tuning them to produce parts with alternating layers of austenite and martensite phase [12]. Thus, strategically designed laser scan patterns can be used to develop specific microstructures by controlling grain orientations and composition in SMAs. With this control of orientations, AM promises to be a useful technique to produce both tailored geometries as well as site-specific property variations.

In anticipation of the advancement of these AM techniques that enable tailoring of the microstructure, we focus on a related mechanistic issue: the influences of the grain structure, grain orientations, and microhole geometry on the deformation localization and crack growth in NiTi SMA. We collectively explore deformation localization and crack growth since most often microcracks nucleate in the regions of accumulated inelastic deformation. Microholes provide a simple model for the complex stress state that is likely to develop in porous SMA assemblies as well as AM-produced SMA products with small-scale features. These mechanistic aspects have been extensively studied individually. For example, microholes and notches are known to induce multiaxial stress states and multiple martensite variants in their vicinity [13] and also to act as the nucleation sites for cracks [14], while grain boundaries are known to constrain deformation due to phase transformation [15]. Crack growth in SMAs has been extensively studied both at the individual crystal scale as well as in a statistical sense, using empirical [16-19] as well as modeling-based techniques $[20,21]$. However, the influences of both structural features and the accompanying microstructure on deformation localization and crack growth have not been studied together.

This work employs a coupled experimental and modeling approach to provide a new understanding of the effects of microstructure and defects on localization and crack growth. We use microstructural modeling to systematically vary the grain orientation in two virtual SMA specimens with microholes to explore the trends in strain localization around holes. We then use an empirical approach to qualitatively characterize the paths followed by cracks growing from microholes and traversing multiple grains in a superelastic NiTi SMA planar specimen loaded in tension. In the Materials, Experiments, and Simulations section, we introduce the modeling and experimental methods used. The first half of the "Results and Discussion" section contains results from the simulations, while the latter half consists of results and discussions from the experimental investigations.

\section{Materials, Experiments, and Simulations}

In this work, we used two methods: cyclic tension experiments with ex situ electron microscopy and micromechanical modeling. The experimental approach was used to monitor crack growth around a pair of microholes in a NiTi plate during cyclic tensile loading. The micromechanical modeling was used to simulate the strain evolution and strain-band formation in virtual samples. The microstructures in these simulations were based on the experimental sample. The details of these methods are described below.

\section{Description of Materials, Specimen, and Experiments}

The superelastic NiTi SMA material containing $55.6 \mathrm{wt} \%$ $\mathrm{Ni}$ and the experimental specimen used here are similar to the ones extensively described in [6]. The relevant details are summarized below.

1. The material is superelastic, polycrystalline $55.6 \mathrm{Ni}-\mathrm{Ti}$ (wt $\%$ ) with an average grain size of $\approx 87 \mu \mathrm{m}$. The map of austenite (cubic) grains and the grain orientation along the loading axis are shown in Fig. 1a.

2. A schematic of the sample geometry is shown in Fig. 1d. The sample gage section is $25 \mathrm{~mm} \times 3 \mathrm{~mm}$ and the gage is $\approx 0.5 \mathrm{~mm}$ thick.

3. In the center of the gage, two holes were drilled into the sample as schematically shown in Fig. 1e (left). The hole diameter was $564 \mu \mathrm{m}$ with a center-to-center spacing of $970 \mu \mathrm{m}$. The hole size and the spacing were chosen to be within one order of magnitude of the mean grain size.

Mechanical testing was done at an engineering strain rate of $\approx 10^{-3} / \mathrm{s}$ using a Sintech $20 \mathrm{G}$ load frame. The sample was loaded in uniaxial tension for 100 load cycles to a nominal axial strain of $1 \%$, as measured by a $10 \mathrm{~mm}$ 
extensometer with knife around the center of the sample gage section. The sample was removed from the load frame after 10, 20, 50, and 100 cycles for imaging of any cracks. The imaging was performed using an FEI Quanta 600F scanning electron microscope (SEM).

\section{Simulations Using a Micromechanics-Based Model}

A microstructural finite element (FE) model for phase transformation by Manchiraju and Anderson was used to perform the simulations [22]. Key features of the model are summarized below.

1. Each element was modeled as an austenite single crystal with three deformation mechanisms:

a. Anisotropic linear elasticity in the austenite and martensite phases.

b. Martensitic phase transformation between austenite (cubic, B2) and martensite (monoclinic, B19') phases at the habit plane variant (plate) scale.

c. Plasticity in austenite in the framework of crystal plasticity. Six $\left\langle\begin{array}{llll}1 & 0 & 0\end{array} /\left\{\begin{array}{lll}1 & 1 & 0\end{array}\right\}\right.$ and six $\left\langle\begin{array}{llll}1 & 0 & 0\end{array}\right\rangle /\left\{\begin{array}{lll}0 & 1\end{array}\right.$ 0\} B2 slip systems were considered.

2. The inputs to the model, reflecting the deformation mechanisms described above, were a. Elasticity: elastic constants and thermal expansion coefficients for the martensite and austenite phases.

b. Phase transformation: equilibrium transformation temperature, latent heat of transformation per unit volume, coefficients of a phenomenological hardening matrix accounting for plate-plate interaction, and the critical energy barrier for austenite to martensite phase transformation (which is assumed to be equal in magnitude but of opposite sign for martensite to austenite reverse transformation).

c. The input also includes the crystallographic orientation at every material point.

3. Based on a prescribed deformation, final outputs of the model are the stress tensor and the martensite plate volume fractions at each integration point in the $\mathrm{FE}$ model.

The goal of this work is to model the transformation characteristics around structural features at relatively low loads. Therefore, we suppressed plasticity by setting the critical resolved shear stress to a very high value (1000 MPa). The equilibrium transformation temperature was set from differential scanning calorimetry (DSC) testing done on the experimental sample, as described in
Fig. 1 Schematic of experimental and virtual specimens. a Orientation map of the experimental sample with holes with $\mathbf{b}$ the color key for orientations with respect to the loading axis. $\mathbf{c}$ The dependence of theoretical axial transformation strain on orientation, based on calculations from the crystallographic theory of martensite [23]. d Schematic of the experimental specimen with arrows showing the loading direction. e Sample geometry and boundary conditions used in the simulations. The left and right panels show the hole structure in Simulations 1 and 2, respectively, while the central panel shows the mesh density used in Simulation 2 (Color figure online) (a)



(b)

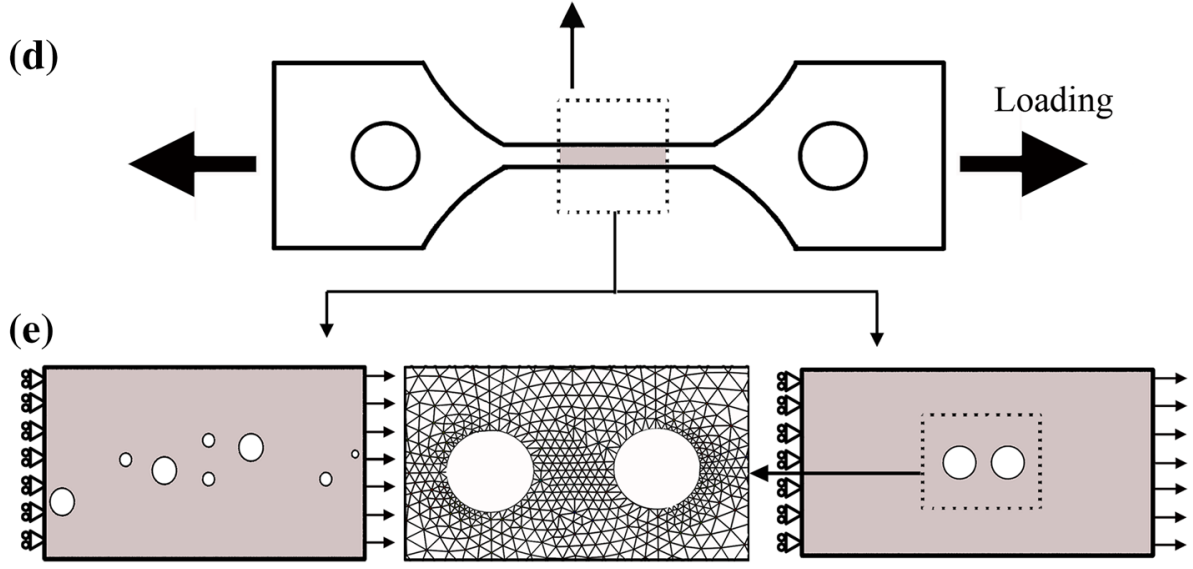


[6]. Representative values for other material parameters in the simulations are listed in Table 1 in [22].

Since a majority of deformation of similar samples in [6] are observed to be concentrated around the holes, a rectangular region localized around holes was modeled using Abaqus FE software. First, the average grain size from Fig. 1a was used to tessellate the model with grains. The grains in the model were assumed to be columnar through the thickness. Second, a mesh comprising tetrahedral elements (C3D10 $\mathrm{M}$ in Abaqus notation, ten integration points, bilinear interpolation) was created. Finally, every grain was assigned a particular orientation, and all elements located within a tessellated grain were identified and assigned that same crystallographic orientation to simulate a polycrystal specimen. The mesh was created such that it was fine $(\approx 225$ elements per grain) near the holes where most of the deformation was concentrated and coarser (up to 5 elements per grain) away from the holes, as shown in Fig. 1e (center schematic). One end of the rectangular model was held stationary, while a constant displacement (at a similar rate to the experimental loading) was applied on the other edge to simulate uniaxial tensile loading.

Using this model setup, two simulations with the same model geometry described above, but with a different hole geometry, as shown in Fig. 1e, were considered to model the interactions between structure and microstructure.

Simulation 1 A $25 \mathrm{~mm} \times 3 \mathrm{~mm} \times 0.25 \mathrm{~mm}$ gage section with a random grain orientation distribution was used in this simulation. This distribution resembles actual porous materials in polycrystals by including eight holes of varying sizes $(100,200$, and $400 \mu \mathrm{m})$ and separations arranged in a staggered manner through the central part of the specimen, as shown in Fig. 1e (right schematic). A tessellation was performed on the surface of the specimen, using the average grain size obtained from Electron BackScatter Diffraction (EBSD) and extrapolated through the thickness of the sample assuming through-thickness grains.

Simulation 2 This simulation consists of only two holes in the model as a highly idealized representation of a porous SMA specimen. The model in this case represents the same $25 \mathrm{~mm} \times 3 \mathrm{~mm} \times 0.25 \mathrm{~mm}$ gage section as in the above case. The diameter of each hole is $150 \mu \mathrm{m}$, and the center-to-center hole separation is $300 \mu \mathrm{m}$ (Fig. 1e, left schematic). Two separate instances of this case were considered. In the first instance, a specimen with a random orientation distribution was used to mimic a polycrystal with random texture. In the second instance, the orientation of selected grains above one of the holes was changed so that the resultant grain orientations highly favored phase transformation in the loading direction. These two instances are described further in the next section in the discussion of Fig. 3.

\section{Results and Discussion}

\section{Simulation: Structural Features Determine the Initiation Sites and Grain Orientations Determine the Continuity of High-Strain Bands}

Figure 2 shows the simulated axial strain around holes at four different nominal strain states in Simulation 1 described in the previous section. While the nucleation of large-strain bands begins directly at the sites of stress concentration, above and below the holes, the geometry of these strain bands itself is more complex. Figure 2a shows the initial load-free state and Fig. 2b-d show the propagation of the high-strain bands in the regions around the holes. The sequence of strain states in Fig. $2 b-d$ shows tortuous, asymmetric, and occasionally discontinuous growth of strain bands with increasing loads. The grayscale contour indicates the simulated axial strain, while the colored insets show the theoretical axial transformation strain inside the box in the gray contours. The theoretical strain depends on the grain orientation as indicated in Fig. 1c.

The location of strain bands in a "V" shape above and below the holes in the simulation is expected based on the theory of elasticity. Those sites are the locations of stress maxima. The observed complex geometry in the growth of strain bands can be rationalized based on the favorability for transformation of the grains that these bands encounter. The insets in Fig. 2b-d use the Crystallographic Theory of Martensite to show the theoretical axial transformation strain that individual grains can produce [23]. Dependence of theoretical axial transformation strain on the orientation is shown in Fig. 1c; generally, in NiTi, grains with $\left[\begin{array}{lll}1 & 1 & 1\end{array}\right]$ and $\left[\begin{array}{lll}1 & 1 & 0\end{array}\right]$ directions oriented with the loading direction produce a larger transformation strain and are therefore easier to transform compared to [ $\left[\begin{array}{lll}1 & 0 & 0\end{array}\right]$ oriented grains. The black arrows in the insets in Fig. $2 \mathrm{~b}-\mathrm{c}$ indicate the direction of propagation of the bands. When a propagating band encounters a highly unfavorable grain, there are two possible outcomes. As seen in Fig. 2d, if the grain is small enough, the band can grow around the grain and continue propagating, as indicated by the ellipse just below the hole; however, if the grain is large enough, it completely stops the growth of the band in that direction, as shown by the ellipse near the bottom left of the hole. In this latter case, we have observed that a different strain band typically nucleates and grows from nearby, in a different direction, based on an efficient pathway in its neighborhood. Thus, branching strain bands can be seen in the lower-right portion of Fig. 2d. A similar observation was made by Richards et al. [24], who observed phase transformation spreading between favorably oriented grains with slip 
acting as a bridge in unfavorably oriented grains for phase transformation.

\section{Simulation: Local Orientations can be Tailored to Relieve Stress Concentrations}

The results in the previous section demonstrate that in SMA specimens where the pore size is comparable to the

(a)

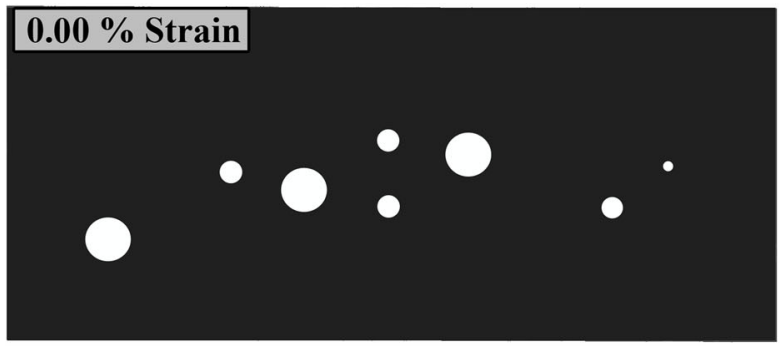

0.0 Simulated Axial Strain (\%)

(c)

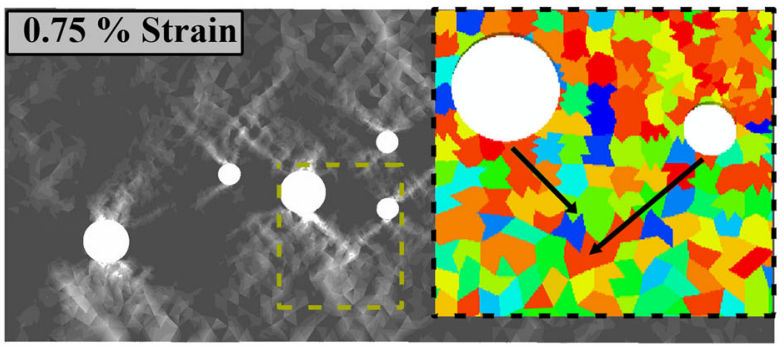

Fig. 2 Simulated estimates (grayscale contours) of axial strain from Simulation 1, with the theoretical axial transformation strain for the boxed region indicated by the colored plots. a The entire specimen in the initial load-free state. Grayscale contours in (b-d) show different snapshots of a at increasing nominal macrostrains: $\mathbf{b}$ at $0.6 \%$, $\mathbf{c}$ at $0.75 \%$, and $\mathbf{d}$ at $0.9 \%$. The colored insets in each subfigure (bd) show the theoretical axial transformation strain for the boxed grain size, the stress concentration and the growth of strain bands can be heterogeneous. Thus, site-specific control of microstructural features such as crystallographic orientation can be used to alter stress states in specific regions of the sample. This section shows a specific example of this idea.

Figure 3a shows the simulated axial stress at $1 \%$ macrostrain in Simulation 2, when the grain orientations (b)



3.5 Theoretical Axial Strain (\%) 6.5

(d)

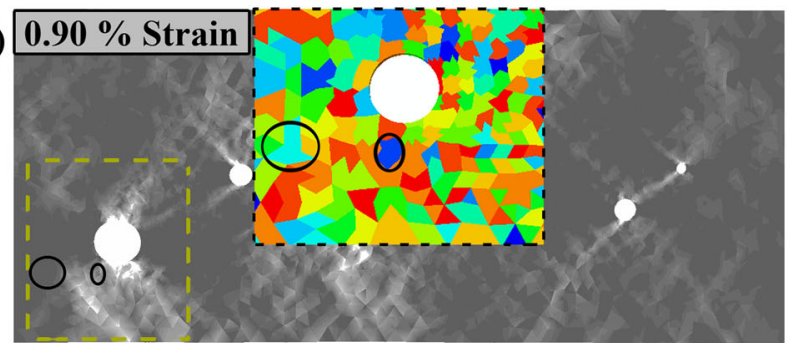

region similar to that shown in (a). The dependence of theoretical transformation strain on orientation is shown earlier in Fig. 1d. The loading in all subfigures is along the horizontal direction. The black arrows in the insets show the direction of propagation of strain bands from nucleation spots. The ellipses in (d) indicate where high-strain bands either completely stopped or grew around unfavorably oriented grains (Color figure online)



xial Stress $(\mathrm{MPa})$



Fig. 3 Results from Simulation 2 showing stress relief near holes when grain orientations are changed. The gray-scale contour shows the axial stress from simulations, while the colored contour is the theoretical transformation strain, which depends on the orientation according to Fig. 1c. In (a), The stress maximum in the sample corresponds to a grain producing relatively small theoretical axial transformation strain, is situated above the hole (inset, blue color with white border), due to inefficient transformation. In (b), when the orientation of that grain is changed to favor transformation (inset, red color with white border), the stress is relieved as shown above the hole by the dotted box (Color figure online) 
are randomly assigned, and the average grain size is obtained from the experimental sample. There is a stress concentration above the hole in a region where an unfavorably oriented grain is located, demarcated by a white outline and also observed in the grayscale axial stress map. The inset shows theoretical axial transformation strain for the grains. The stress concentration site coincides with the location of the grain that produces $3.5 \%$ axial transformation strain. In the second instance of the simulation, the results of which are shown in Fig. 3b, the crystallographic orientation of this grain is changed so that it is now favored for transformation, producing $6.5 \%$ axial transformation strain. Therefore, in case (b), after switching the orientation, the grain transforms easily and the stress concentrations above the hole in (b) are relieved. Tailored material design to proactively target grain orientations in regions of anticipated local stress concentrations might have favorable influence on the cyclic loading performance of porous SMA components. This is a concept that merits further study to advance understanding of the effect of such tailored microstructures. In steels-materials that deform by plasticity—change in pore shape from a circle to an "S" shape increased the fatigue life due to reduction of local stresses [25]. In SMAs, however, the promotion of local transformation to relieve stresses as demonstrated in this
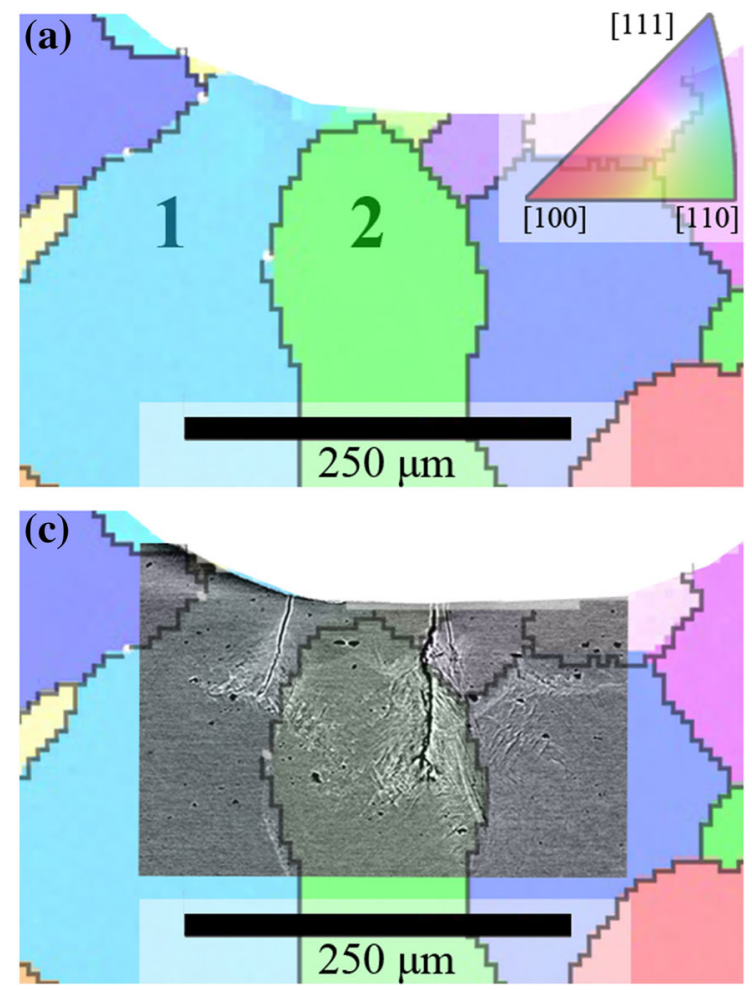

Fig. 4 Crack growth in the bottom left quadrant indicated by the dotted box in Fig. 1a. a Grain orientation with respect to the leading axis. The standard coloring scheme, as shown in the key in Fig. 1b is used. Crack geometry, as seen using ex situ SEM, is superimposed on section may also lead to local transformation-induced slip, leading to an accumulation of defect content over many cycles, which can lead to functional fatigue.

\section{Experimental: Stress Concentration Determines Crack Nucleation and Grain Structure Influences Crack Growth}

The previous subsections used a simulation-based approach to explore the influence of grain orientation on strain localization and a way to reduce local stress by orientation engineering. In this section, we use an experimental approach to show how orientations also influence crack growth. These results are from the investigation of crack growth over 100 cycles up to a nominal axial strain of $1 \%$, as described in the subsection entitled "Description of Materials, Specimen, and Experiments."

Figure 4a shows the EBSD grain maps with SEM images of crack growth superimposed on them (Fig. 4b-d) for the experimental sample after 20,50, and 100 cycles. The region shown in this figure is coincident with the bottomleft quadrant of Fig. 1a, shown by the dotted box. In this region, two cracks are visible: one in the cyan-colored grain (numbered 1) and another in the green-colored grain just to the right (numbered 2). The crack through Grain 1
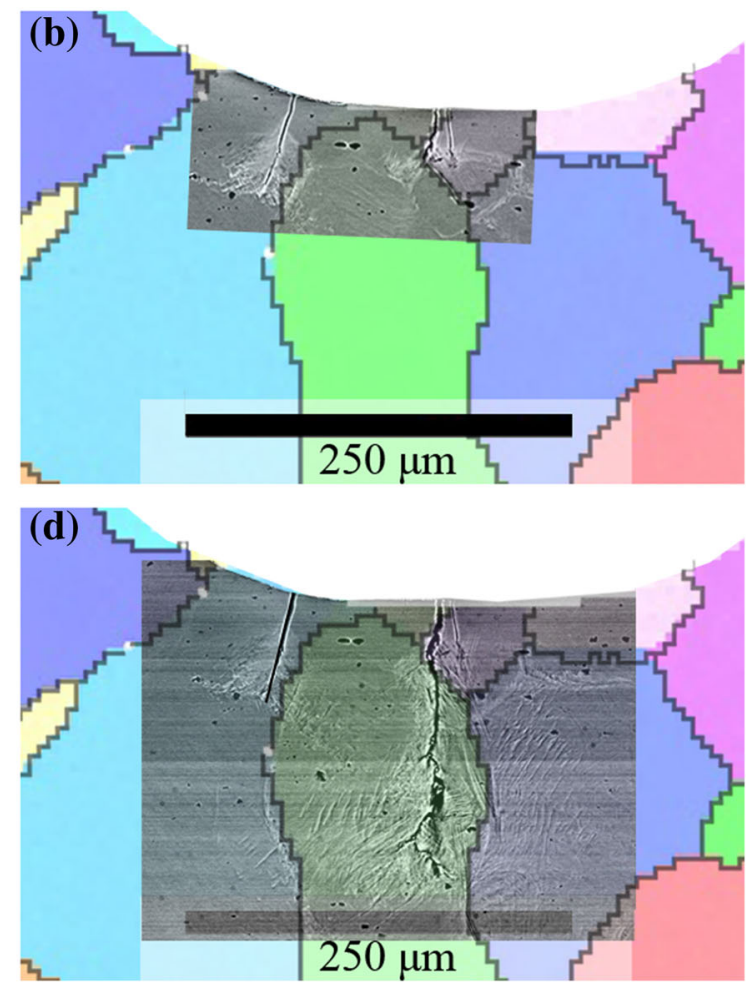

the grain orientation map after b 20 cycles, c 50 cycles, and d 100 cycles. The crack in cyan grain, numbered 1 , does not grow. The crack in green grain (numbered 2) grows; however, it encounters inclusions (dark spots) (Color figure online) 



Fig. 5 Crack growth in the top left quadrant shown by the dotted box in Fig. 1a. a Grain orientation with respect to the leading axis. The standard coloring scheme is used, as shown in the key in Fig. 1b. Crack geometry, as seen using ex situ SEM after b 20 cycles, c 50 cycles, and d 100 cycles. The crack in the aquamarine grain

(numbered 1) grows through a straight path through the entire grain until it reaches a grain boundary, and then it continues to grow into the green grain (numbered 2). The arrows in (d) indicate the change in angle of the crack as it grows from Grain 1 to Grain 2. Residual deformation is seen around the crack (Color figure online)

does not show significant growth during cycling. With an

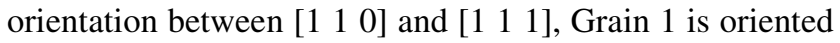
favorably for phase transformation and slip in the austenite phase under uniaxial stress along the horizontal direction. Thus, it is plausible that the stress state is more relaxed under peak load due to profuse phase transformation, stopping crack growth. On the other hand, the crack through Grain 2 grows continuously during cycling, interrupted by small, black inclusion particles and following a tortuous path. In both cases, a band-shaped deformation structure is visible around the cracks.

Figure 5 shows the crack growth observed in the dotted box in the top-left quadrant of Fig. 1a, at the end of 20, 50, and 100 cycles. In this case, one major crack in the center region grew through the aquamarine-colored grain (numbered 1) into the green grain (numbered 2). The crack followed an approximately straight path through Grain 1 at an angle of $\approx 75^{\circ}$ to the loading axis, and then through Grain 2 , at an angle of $\approx 60^{\circ}$ to the loading axis. These angles correspond to $\left\{\begin{array}{lll}1 & 1 & 0\end{array}\right\}$-type planes in the respective grains. Gall et al. [26] showed that fracture in NiTi takes place through cleavage along $\left\{\begin{array}{lll}1 & 0 & 0\end{array}\right\}$ and $\left\{\begin{array}{lll}1 & 1 & 0\end{array}\right\}$ planes. The straight nature of the crack growth suggests crystallographic fracture along a $\left\{\begin{array}{lll}1 & 1 & 0\end{array}\right\}$-type plane. This

observation is in contrast with the previous result, where the crack follows a tortuous path that we postulate is due to the presence of inclusions in its path in that case, as seen by Vaidyanathan et al. [27].

To the left of the hole in Fig. 5 are red grains, which, according to Fig. 1b, are close to a $\left[\begin{array}{lll}1 & 0 & 0\end{array}\right]$ orientation from the loading axis. According to Fig. 1c, such grains are characterized by a high transformation stress and low transformation strain (inefficient for transformation). Since this loading was conducted at relatively low loads (up to a peak nominal strain of $1 \%$ ), it is not expected to show significant deformation due to the high activation threshold for transformation. This is confirmed by the SEM images, by a marked absence of a remnant deformation field around that region seen around cracks in Figs. 4 and 5.

We also monitored the crack growth around the right hole in the experimental sample. Three cracks were observed close to grain boundaries and did not show significant growth over cycling while one large crack primarily grew through a large [lll 110$]$-type grain. These observations are largely consistent with the results presented for the left hole. However, a statistical correlation between grain orientation and propensity for crack growth cannot be made from these observations that considered a 
limited number of grains and two holes of fixed size. A more comprehensive program relating crack growth to grain orientations may follow the approach recently used to relate grain size and fracture resistance [28, 29]. This would involve tracking a larger number of cracks around a broader distribution of pore sizes, grains, and locationsfor example, in a sample similar to the virtual specimen in Simulation 1.

\section{Conclusions}

The goal of this work was to explore two mechanistic phenomena in a representation of porous NiTi shape memory alloys. The first phenomenon, strain localization around holes, was studied using a microstructural modeling approach. Relation between crack growth and grain orientation around holes was explored experimentally. The major conclusions are as follows:

1. The relation between hole location, grain orientation, and strain-band formation is intuitive. Strain bands form at stress concentration sites around holes. However, their growth is enhanced by grains optimally oriented for transformation and impeded by unfavorably oriented grains. Thus, the strain bands can fluctuate in strain and follow tortuous paths.

2. This strain-band geometry's dependence on the orientations can be used to relieve stress concentrations by introducing easy-to-transform grains in the path of the bands. Recent AM techniques may be able to achieve such orientation tailoring.

3. The presence of large [ [ 110$]$-type grains with respect to the loading axis is seen to provide the least resistance to crack growth in areas of stress concentration around the holes.

This work opens an avenue for engineering local crystal orientations to improve fatigue life of components made of materials that are highly anisotropic and heterogeneous in deformation. In the future, it will be interesting to experimentally explore a larger crack population to statistically uncover significant trends between the nature of crack growth and grain orientation, and a more comprehensive picture of polycrystalline fracture in porous shape memory materials. For reducing stress concentration around pores, a future additive manufacturing effort may develop a laserscan strategy that actually realizes the orientation changes discussed in the subsection entitled "Simulation: Local Orientations can be Tailored to Relieve Stress Concentrations."

Acknowledgements The authors would like to acknowledge the use of NUANCE (NSF ECCS-1542205), MatCI and CLAMMP (NSF
DMR-1121262) facilities at Northwestern University for electron microscopy and mechanical testing and the computing facilities at XSEDE (Extreme Science and Engineering Discovery Environment, NSF ACI-1053575). Financial support for this work was provided by the Department of Energy, Office of Basic Energy Science (Grant No. DE-SC0010594). The authors thank Dr. Sivom Manchiraju (formerly at The Ohio State University) for making available the Abaqus UMAT code used in this work, and Ms. Amy Brice (Colorado School of Mines) for helping with proof-reading and formatting of the manuscript.

\section{References}

1. Bansiddhi A, Sargeant TD, Stupp SI, Dunand DC (2008) Porous NiTi for bone implants: a review. Acta Biomater 4(4):773-782

2. Hartl DJ, Lagoudas DC (2007) Aerospace applications of shape memory alloys. Proc Inst Mech Eng Part G 221(4):535-552

3. Bram M, Köhl M, Buchkremer HP, Stöver D (2011) Mechanical properties of highly porous NiTi alloys. J Mater Eng Perform 20(4-5):522-528

4. Dadbakhsh S, Speirs M, Van Humbeeck J, Kruth J-P (2016) Laser additive manufacturing of bulk and porous shape-memory NiTi alloys: From processes to potential biomedical applications. MRS Bull 41:765-774

5. Elahinia M, Moghaddam NS, Andani MT, Amerinatanzi A, Bimber BA, Hamilton RF (2016) Fabrication of NiTi through additive manufacturing: a review. Prog Mater Sci 83:630-663

6. Paul PP, Paranjape HM, Amin-Ahmadi B, Stebner AP, Dunand DC, Brinson LC (2017) Effect of machined feature size relative to the microstructural size on the superelastic performance in polycrystalline NiTi shape memory alloys. Mater Sci Eng A 706:227-235

7. Kobryn PA, Semiatin SL (2001) The laser additive manufacture of Ti-6Al-4V. JOM 53:40-42

8. Collins P, Brice D, Samimi P, Ghamarian I, Fraser H (2016) Microstructural control of additively manufactured metallic materials. Annu Rev Mater Res 46:63-91

9. Dehoff RR, Kirka MM, Sames WJ, Bilheux H, Tremsin AS, Lowe LE, Babu SS (2015) Site specific control of crystallographic grain orientation through electron beam additive manufacturing. Mater Sci Tech 31:931-938

10. Saedi S, Turabi AS, Andani MT, Moghaddam NS, Elahinia M, Karaca HE (2017) Texture, aging, and superelasticity of selective laser melting fabricated Ni-rich NiTi alloys. Mater Sci Eng A 686:1-10

11. Franco BE, Ma J, Loveall B, Tapia GA, Karayagiz K, Liu J, Elwany A, Arroyave R, Karaman I (2017) A sensory material approach for reducing variability in additively manufactured metal parts. Sci Rep 7:3604

12. Wang X, Speirs M, Kustov S, Vrancken B, Li X, Kruth J-P, Van Humbeeck J (2018) Selective laser melting produced layerstructured NiTi shape memory alloys with high damping properties and elinvar effect. Scr Mater 146:246-250

13. Niendorf T, Lackmann J, Gorny B, Maier H (2011) In situ characterization of martensite variant formation in nickel-titanium shape memory alloy under biaxial loading. Scr Mater 65:915-918

14. Kohl M, Bram M, Moser A, Buchkremer H, Beck T, Stover D (2011) Characterization of porous, net-shaped NiTi alloy regarding its damping and energy-absorbing capacity. Mater Sci Eng A 528:2454-2462

15. Carroll JD, Abuzaid WZ, Lambros J, Sehitoglu H (2013) On the interactions between strain accumulation, microstructure, and fatigue crack behavior. Int J Fract 180:223-241 
16. Creuziger A, Bartol LJ, Gall K, Crone WC (2008) Fracture in single crystal NiTi. J Mech Phys Solids 56:2896-2905

17. Wu Y, Ojha A, Patriarca L, Sehitoglu H (2015) Fatigue crack growth fundamentals in shape memory alloys. Shape Mem Superelast 1:18-40

18. Daymond MR, Young ML, Almer JD, Dunand DC (2007) Strain and texture evolution during mechanical loading of a crack tip in martensitic shape-memory NiTi. Acta Mater 55:3929-3942

19. Robertson SW, Mehta A, Pelton AR, Ritchie RO (2007) Evolution of crack-tip transformation zones in superelastic nitinol subjected to in situ fatigue: a fracture mechanics and synchrotron X-ray micro diffraction analysis. Acta Mater 55(18):6198-6207

20. Jape S, Baxevanis T, Lagoudas DC (2017) On the fracture toughness and stable crack growth in shape memory alloy actuators in the presence of transformation-induced plasticity. Int $\mathbf{J}$ Fract 209(1-2):117-130

21. Baxevanis T, Lagoudas DC (2015) Fracture mechanics of shape memory alloys: review and perspectives. Int J Fract 191:191-213

22. Manchiraju S, Anderson PM (2010) Coupling between martensitic phase transformations and plasticity: a microstructure-based finite element model. Int J Plast 26:1508-1526
23. Bhattacharya K (2003) Microstructure of martensite: why it forms and how it gives rise to the shape-memory effect. Oxford University Press, Oxford

24. Richards AW, Lebensohn RA, Bhattacharya K (2013) Interplay of martensitic phase transformation and plastic slip in polycrystals. Acta Mater 61:4384-4397

25. Javid F, Liu J, Rafsanjani A, Schaenzer M, Pham MQ, Backman D, Yandt S, Innes MC, Booth-Morrison C, Gerendas M, Scarinci T, Shanian A, Bertoldi K (2017) On the design of porous structures with enhanced fatigue life. Ext Mech Lett 16:13-17

26. Gall K, Yang N, Sehitoglu H, Chumlyakov YI (2001) Fracture of precipitated NiTi shape memory alloys. Int J Fract 109:189-207

27. Vaidyanathan R, Dunand DC, Ramamurty U (2000) Fatigue crack-growth in shape-memory NiTi and NiTi-TiC composites. Mater Sci Eng A 289:208-216

28. Ahadi A, Sun Q (2016) Grain size dependence of fracture toughness and crack-growth resistance of superelastic NiTi. Scr Mater 113:171-175

29. LePage WS, Ahadi A, Lenthe WC, Sun Q, Pollock TM, Shaw JA, Daly SH (2017) Grain size effects on NiTi shape memory alloy fatigue crack growth. J Mater Res 33(2):91-107 